\title{
Performance Evaluation and Design for Variable Threshold Alarm Systems through Semi-Markov Process
}

\begin{abstract}
In large industrial systems, alarm management is one of the most important issues to improve the safety and efficiency of systems in practice. Operators of such systems often have to deal with a numerous number of simultaneous alarms. Different kinds of thresholding or filtration are applied to decrease alarm nuisance and improve performance indices, such as Averaged Alarm Delay (ADD), Missed Alarm and False Alarm Rates (MAR and FAR). Among thresholdbased approaches, variable thresholding methods are well-known for reducing the alarm nuisance and improving the performance of the alarm system. However, the literature suffers from the lack of an appropriate method to assess performance parameters of Variable Threshold Alarm Systems (VTASs). This study introduces two types of variable thresholding and proposes a novel approach for performance assessment of VTASs using Priority-AND gate and semiMarkov process. Application of semi-Markov process allows the proposed approach to consider industrial measurements with non-Gaussian distributions. In addition, the paper provides a genetic algorithm based optimized design process for optimal parameter setting to improve performance indices. The effectiveness of the proposed approach is illustrated via three numerical examples and through a comparison with previous studies.
\end{abstract}

Index Terms - Alarm management; Industrial alarm systems; Variable threshold alarm system; Performance assessment; Semi-Markov Process; Optimal thresholding.

\section{INTRODUCTION}

Nowadays, in large industrial systems, enormous number of sensors are installed in different parts of the systems for online condition monitoring of the plant during operation. By using these sensors, abnormal behaviors of systems are detectable. Whenever a process variable exceeds a certain threshold, an alarm is raised (in auditory or visual form) to indicate an unexpected behaviour of the system that can be due to fault occurrence in sub-systems, malfunctioning sensors, or any other related reason [1]. An alarm system receives data from the sensors and process them to delineate abnormalities for operators. Therefore, just like pain in human body, these systems generate important signs of system's abnormality. In modern industrial plants, alarm systems play a vital role in ensuring the process safety and efficiency. However, an immense number of potential alarms that can be raised simultaneously make the alarm management task challenging for any industry $[2,3]$. For example, during the catastrophic accident in the nuclear power plant at Three Mile Island in 1979, the worst nuclear accident in the US history, operators faced challenging situation due to redundant and confusing information provided to them, much of the information provided was irrelevant and illusory during the accident $[2,4]$.

Different activities, tools and procedures have been developed to improve the performance and effectiveness of alarm systems and some standards such as EEMUA-191 and ISA-18.2 are defined [5,6]. Averaged Alarm Delay (AAD), Missed Alarm Rate or Probability (MAR/MAP), False Alarm Rate or Probability (FAR/FAP) and Chattering are four specified indices that can be used for an alarm system's performance assessment. Interested readers are referred to [7] for further details about performance indices. However, there are still some ongoing research works to improve the performance of alarm systems, and a brief review of research works related to this area is studied as follows:

\section{A Brief Literature Review on Performance Assessment of Alarm Systems}

The existing standards, guidelines and regulations for alarm systems and their design procedure have been reviewed by [8]. Regarding the alarm systems, the study highlighted the challenges and opportunities. A strategy for Integration of alarm system implementation and operational practices has been proposed and the potential use of cyber-physical systems in alarm systems and industrial automation has been suggested. The latest trends and developments in fire alarms have been analyzed by [9]. The study pointed out that most false alarms were the result of poor installation, imperfect maintenance, and human factors. A critical view on current and potential issues and challenges has been given in this research. Reference [10] focused on operation metrics, visualization plots and alarm floods as decision support tools for alarm systems. The paper introduced multi-layered radar plots to increase the readability of alarm 
metrics. A ranking method is also proposed by this paper to reorder design procedure based on alarm-flood criticality index. The state-of-the-art regarding data-driven multi-mode process modelling and monitoring has been provided by [11]. They reviewed existing methodologies of modelling and monitoring for multi-mode processes. A systematic clustering and characterization for both modelling methods and monitoring approaches have been studied as well.Different approaches for improvement of performance in alarm systems and detract alarm persecution have been investigated in [12], among studied solutions filtering approaches, delay timers and deadband can be highlighted. However, in these ways, some delay occurs during alarm detection. In [1], trade-offs between the time delay to raise an alarm and the rate of missed and false alarm has been studied through applying a novel filtering approach for alarm systems. This paper introduced exponentially weighted moving average (EWMA) filter. Another technique with receiver operating characteristic-based curve for designing deadbands or thresholds to reduce FAR and MAR has been proposed in [13].

In [7], FAR, MAR, and AAD were considered as performance metrics to design and assess the performance of univariate alarm systems. In that approach, a simple threshold-based method called mean change detection was proposed to distinguish the normal alarms from the abnormal ones. However, the application of this approach is limited to a Gaussian distribution. Cheng et al. [14] introduced an optimal filter design method to enhance the performance of alarm system over the classical moving average filters. A new visualization for illustrating the time-lagged correlation between different alarms has been proposed using the correlation color map by [15]. Adnan et al. [2] introduced a new method to improve conventional delay timer methods, which was called generalized delay timers. Also, FAR, MAR, and Expected Detection Delay (EDD) were calculated using a Markov model to evaluate the performance of this approach in comparison with a conventional delay timer. In [16], the authors presented a method to minimize missed alarm probability (MAP) and false alarm probability (FAP) in a multidimensional space by optimizing multivariable alarm threshold. Although this approach could reduce the number of missed alarms, the reduction of both false alarm and missed alarm simultaneously is impossible. In [4], an improved version of delay timer has been introduced, which was based on multiple orders and set-points to cover transition behavior between non-alarm and alarm mode.

In [17], a method for multivariate alarm thresholds optimization by combining FAP, MAP, and correlation analysis was presented to solve an alarm threshold optimization problem to reduce nuisance alarms. An evidence theory based online variable threshold method was introduced in [18] to take into account the uncertainties associated with the process variables while optimizing the design of alarm systems. Gao et al. [19] proposed a correlation consistencybased multivariate alarm thresholds optimization. In this paper, Pearson correlation analysis is applied to obtain the correlation coefficients of process data, and Particle Swarm Optimization (PSO) algorithm is used for the problem optimization. To improve the efficiency of existing thresholding-based alarm system, mean-change point detection method was utilized to propose an extended adaptive threshold alarm system in [20]. A penalty-based approach for delay timer design has been introduced in [21] and formalized in [22]. This approach to designing an alarm system was an extension to the $n$-sample delay timer and they proved that their approach has better effectiveness in terms of MAR, FAR and AAD. They also presented a new Mean Time to Alarm (MTTA) parameter for measuring the system's delay with less computational effort. A novel linear filter has been designed based on a stochastic model of the alarm system by [23] to reduce the probability of false and missed alarms. This study has been implemented on V94.2 gas turbine.

The analysis of multimode delay-timers based on Hidden Markov Model (HMM) has been proposed in [24]. This paper used the particle swarm optimization to minimize FAR, MAR and EED that were obtained from HMM. A Markov process-based performance assessment of time-deadband univariate alarm system has been studied by [25]. Reference [26] introduced a multi-set-point delay timer alarm configuration. This paper provided new Markov models of FAR, MAR and AAD to assess their performance. The application of Deep Learning in Natural Language Processing (NLP) is illustrated on Alarm log information with capability of alarm prediction by [27]. Reference [28] proposed a median filters-based method for chattering reduction in industrial plants. The proposed method is capable of satisfying false alarm and missed alarm rates' constraints.

Although a significant number of researches have been performed so far, to the best of the authors' knowledge, no published work has considered the issue of performance analysis of VTAS by using the concept of Priority-AND (PAND) gate [29] and semi-Markov process (SMP). This study introduces two types of VTAS (i.e. variable threshold and variable threshold with variable deadband), which are capable of reducing alarm nuisance and improving performance. Afterwards, a novel methodology is proposed by utilizing the functional behavior of a PAND gate for the MAR and FAR evaluation of VTAS in different conditions. The PAND gate models of the FAR and MAR are solved by creating semi-Markov models of them. The results are then validated through Monte Carlo simulation and 
compared with some other existing research works. The application of semi-Markov process allows the proposed approach to work with non-Gaussian distributions. Moreover, to facilitate optimal design of alarm systems, the optimal parameters were obtained and analyzed using the Genetic algorithm [30]. This systematic approach and its solution enable alarm systems designers to have a deeper view on performance behavior of these systems.

\section{B. Contributions of the Paper}

- The first and most important contribution of this paper is to propose an approach for performance evaluation of complex alarm systems such as adaptive threshold alarm systems, neural networks and Kalman filters [31]. In other words, the proposed approach deals with the evaluation of probabilistic key performance indices such as the probability of false alarm, and the probability of missed alarm. This approach is an answer for the research question of how can we evaluate the probabilistic key performance indices of complex alarm systems with variable thresholds? It should be noted that traditional approaches like confusion matrix is not comprehensive as this approach is.

- By the use of Semi-Markov Process for the first time in the alarm management research domain, we are able to evaluate probabilistic key performance indices of the complex alarm systems with non-gaussian probability density functions. We also provide alternative probabilistic expressions to deal with non-parametric and numerical probability density function. To prove the validity of the proposed models, a comparison with Monte Carlo Simulation is provided.

- Metaheuristic optimization is used to create an optimized design of adaptive alarm systems. In fact, the proposed SMP has been embedded in the cost function, a multi-objective constraint Genetic Algorithm (GA) to regulate the parameters of adaptive threshold and minimize the probability of missed alarm and probability of false alarm.

- Considering the importance of alarm prioritization in the alarm management domain, a simple priority calculation based on the probability of missed alarm and probability of false alarm has been recommended.

- Using Priority-AND (PAND) gate is a starting point to use the concept of dynamic fault tree and its conversion to a dynamic alarm tree. This PAND gate is a hint to the future research of how a dynamic top-down deductive model can represent the alarms and their dynamic combinations such as sequence, and delay.

- The applicability and usability of the proposed method have been illustrated by an industrial implementation. The V94-2 Gas Turbine is a complex industrial case study that has been used to prove the industrialization of the proposed approach.

\section{The Paper Organization}

The rest of paper is organized as follows. Next section describes problem statement and definitions. Section III introduces the threshold-based alarm systems. In section IV, for performance assessment of alarm systems, a novel methodology based on PAND gate and SMP is presented. Section V evaluates the efficiency of the proposed approach through numerical examples and comparison with other existing works. The penultimate section outlines the strengths and weaknesses of the proposed method. Finally, section VII concludes the paper with concluding remarks and direction for future research.

\section{PROBLEM STATEMENT AND DEFINITIONS}

The performance of an alarm system is mainly subjected to three parameters: rate of false and missed alarm and mean alarm delay. For this purpose, consider the random discrete signal $x(t)$ as the measurement of a process variable with sampling time $h$ and its associated alarm trip point $x_{t p}$ as shown in Fig. 1. 


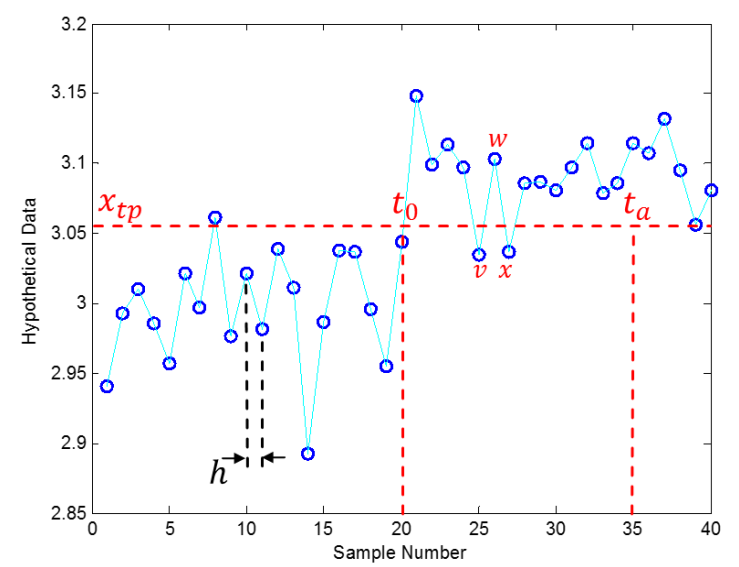

Fig. 1. A random discrete signal $x(t)$ as the measurement of a process variable

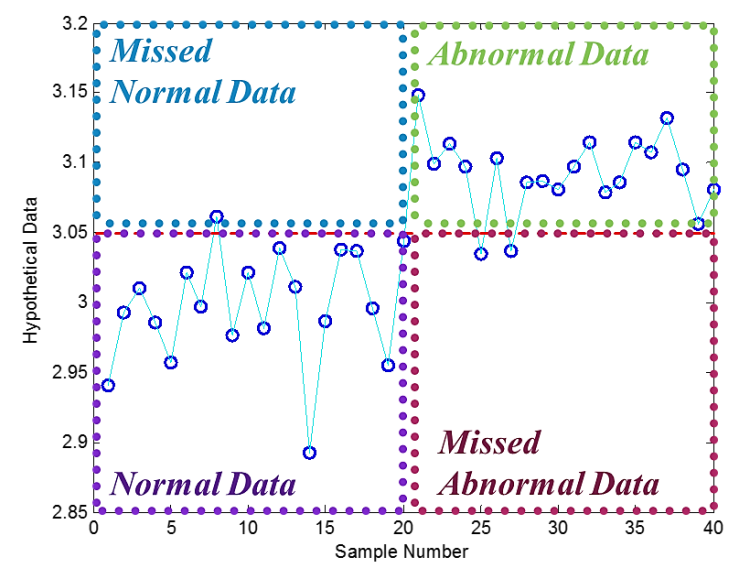

Fig. 2. Classified Normal, Abnormal, Missed Normal (False Alarm) and Missed Abnormal (Missed Alarm) parts of $x(t)$

Due to a well-known method such as on-delay method, in which if $n$ consecutive samples of $x(t)$ exceed the trip point, the alarm is raised. Suppose that normal data are the samples of $x(t)$ that are less than $x_{t p}$ and abnormal data are the samples of $x(t)$ which exceed the $x_{t p}$. Hence, this figure can be categorized into four sections as seen in Fig. 2.

After categorizing normal and abnormal data, the probability density function of them can be obtained as illustrated in Fig. 3. After classifying the measurement values into two normal and abnormal classes, the probability of false alarm and missed alarm can be calculated as follows respectively.

$$
\begin{aligned}
& F A R=q_{1}=\int_{x_{t p}}^{+\infty} q(x) d x \\
& M A R=p_{2}=\int_{-\infty}^{x_{t p}} p(x) d x
\end{aligned}
$$

where $p(x)$ stands for the pdf of abnormal part of measurement and $q(x)$ stands for the pdf of normal part of the measurement. 


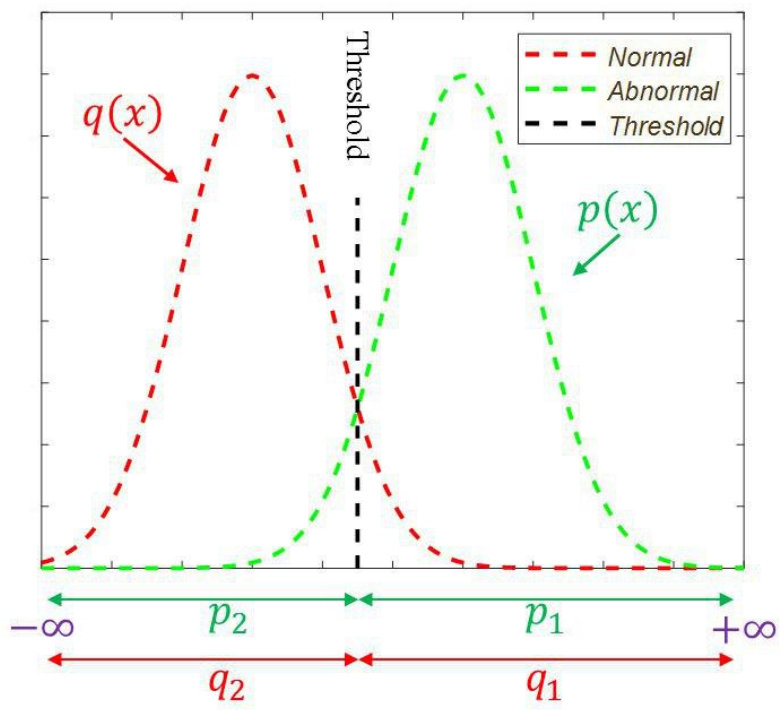

Fig. 3. Separated probability density function of normal and abnormal part of $x(t)$

Now, suppose that the $x(t)$ as a measurement or monitoring signal is changing from the normal condition to abnormal at the time $t_{0}$, and the first sample of $x(t)$ is equal or larger than $x_{t p}$ at the time $t_{a}$. The alarm delay can be defined as follows:

$T_{d}=t_{a}-t_{0}$

Since the signal $x(t)$ is a discrete random variable, the alarm delay $T_{d}$ is a random variable. Hence, the average alarm delay (AAD) is defined as the expected value of $T_{d}$.

$\bar{T}_{d}=E\left(A_{d}\right)$

In general, we need multidimensional joint pdfs of $x\left(t_{0}\right) \cdot x\left(t_{0}+h\right) \cdot \ldots$ to compute $\bar{T}_{d}$. For simplicity, it is assumed that $x(t)$ is independent and identically distributed (IID). Therefore, according to [7], the probability mass function of $T_{d}=i h$ can be written as:

$P\left(T_{d}=i h\right)=p_{2}^{i} p_{1}$

Where, $p_{1}=1-p_{2}$, also the AAD is:

$\bar{T}_{d}=E\left(T_{d}\right)=\sum_{i=0}^{\infty} i h p_{2}^{i} p_{1}=h \frac{p_{2}}{p_{1}}$

\section{BACKGROUND OF THRESHOLDING ALARM SYSTEMS}

In this section, simple and variable threshold alarm systems with and without deadband are introduced briefly. As a definition, an alarm exists when the amount of the data is bigger than the amount of predetermined threshold, and otherwise it clears. In the following, the basic definition of alarm by considering zero mean signal data is presented in (7). To ease the computations it is assumed that the system's malfunctioning occurs as a raising and not intermittent fault and we have only one high limit (adaptive limit) for alarm generation. However, malfunctioning with falling occurrence and their low-level limit can be considered through the proposed method.

$$
\text { Alarm }= \begin{cases}1 & |S|>T \\ 0 & |S|<T\end{cases}
$$

where $\mathrm{T}$ is the threshold and $\mathrm{S}$ is the signal. Note that this signal could be a real signal in signal-based method or residual signal in the model-based method. In the following, the concept of thresholding methods is represented as an example.

Example1: Measurements with Normal Distribution

The process variable is considered as a white Gaussian random process with a mean change at $\mathrm{t}_{0}$, i.e.,

$\left\{N(3,1)\right.$ when $t<t_{0}$

$\left\{N(5,1)\right.$ when $t>t_{0}$

where $t_{0}$ is equal to $70 \mathrm{~s}$ with the sampling period $h=1 \mathrm{~s}$. The length of the data is 140 . 


\subsection{Simple Threshold Alarm Systems}

In this type of systems, a fixed threshold is applied on signal to diagnose alarm status. It can be achieved as follows:

$$
T=m \mp \varphi v
$$

where $\varphi$ is Adjustable Index, $\mathrm{m}$ is the mean value of all data, and $v$ is the variance value of all data in labeled signal. Fig. 4 shows the signal $x(t)$ and applied static threshold and deadband.

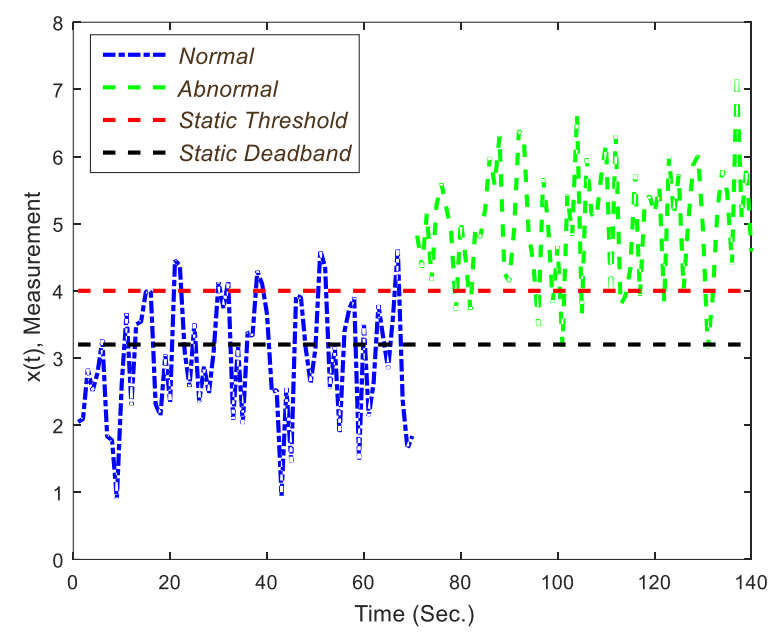

Fig. 4. Generated signal for example 1 with static threshold and deadband.

FAR and MAR as two criteria which are used to analyze the operation of alarm management systems are determined in Table 1.

The proper fixed threshold must be determined to decrease these two criteria as much as possible simultaneously.

When the fixed threshold is big enough, the sensitivity of faults decreases. On the contrary, because of uncertainty and nuisance behavior in a real data, the number of false alarms increases if the static threshold is too low. In this situation, the alarm signals transit between on and off state called Chattering Alarm [32].

Chattering is one of the most significant problems in most industrial control systems, which causes Alarm Flooding in decision-making system. Therefore, finding the solution to this issue is vital. A typical effective method for reduction of alarm chattering is using deadbands [33].

\subsection{Simple Threshold Alarm Systems with deadband}

Deadband method is introduced to improve the performance of alarm management systems in the presence of nuisance data. In fact, deadband can be determined as another limit for clearing alarm in noisy signal [32, 34]. According to threshold type (upper threshold or lower threshold), the suitable deadband is selected.

A deadband is usually defined as a percentage of the threshold or the range of variable [34]. Since the range of the variable is typically unknown, deadband is represented as a proportion of the alarm limit (threshold) in this work.

deadband width(DB) $=\mathrm{L}(1-\mathrm{db})$ for high limit,

$$
\text { deadband width }(\mathrm{DB})=\mathrm{L}(1+\mathrm{db}) \text { for low limit }
$$

where $\mathrm{L}$ is the threshold value, and $d b$ is deadband value.

Furthermore, there are some recommendations for deadband design according to EEMUA standard [5].

In Fig. 4 and in example 1, $\mathrm{L}=4$ and $\mathrm{db}=3.2$ has been considered. Also, FAR and MAR calculated in this way are reported in Table 1 to compare with fixed threshold. It is evident that use of deadband limit makes alarm chattering better than before. However, this way just improves the MAR. It means that fewer missed alarm occurs, but this way is not sufficient to reduce the false alarm.

\subsection{Variable Threshold Alarm Systems}

To reduce the effects of modeling uncertainty and measurement noise, it is vital to apply a larger value of the simple threshold. In fact, this solution can decrease FAR. In this situation, sensitivity can be reduced too. Therefore, this way is not suitable. Moreover, deadband could not reduce FAR as well. So, in order to minimize the false alarm rate, variable thresholds are introduced. The main idea in this kind of threshold is based on varying the threshold value with 
time since disturbances and other uncontrolled effects such as modeling uncertainty and measurement noise changes over the time [35].

While designing a variable threshold, a sliding window with enough length is defined and in each window, mean and variance of data is calculated. It must be noted that window size may vary depending on the trend of the signal. Moreover, momentum factor is used to prevent forgetting the previous samples of data in the variable threshold. In the following Equations ((11) and (12)), calculation process of variables is presented [36].

$$
\begin{aligned}
& \bar{v}=\gamma v(k-1)+(1-\gamma) v(k) \\
& \bar{m}=\gamma m(k-1)+(1-\gamma) m(k) \\
& T(k)=\bar{m}(k) \mp \alpha \bar{v}(k)
\end{aligned}
$$

where $\mathrm{m}$ stands for the average value of data in a normal condition and with the sliding window length, $\gamma$ is momentum factor, $v$ is the variance of data in a normal condition and with the sliding window length, $\mathrm{k}$ is the length of the window (number of samples in each window), $\alpha$ is an adjustable factor, and $\mathrm{T}$ is variable threshold value in the $\mathrm{k}^{\text {th }}$ window [20].

The main problem here is to choose the length of the time window $n$ appropriately. If $n$ is selected too small, the threshold adapts very quickly to any change in the residual caused by any factor, e.g. disturbances, noise or a fault. If $n$ is too large, the threshold acts in a similar way as a constant one, and the sensitivity of decision-making is decreased [36].

Also, some assumptions are considered to apply the variable threshold on signals. In this paper, it is assumed that normal condition of the signal is available and variable threshold can be applied to the signal in this situation. After that, determined variable threshold is applied on the signal in the real situation for decision-making. Lets consider mentioned assumptions, twenty five for the length of window, $\gamma=0.5$. Then probability density function of data in both abnormal and normal condition after applying adaptive threshold can be shown in Fig. 6 (without considering deadband pdf). Note that, as the data of variable threshold have mean and variance, so for these data, pdf is considered. Also, FAR and MAR calculated in this way are reported in Table 1 for comparison with fixed threshold.

By comparing the FAR and MAR calculated by this method with two previous methods, it is clear that the value of false alarm rate is reduced less than the other methods. However, in comparison with method 2 (simple threshold with deadband), there is no improvement in MAR reduction. To overcome this drawback, we introduce variable threshold with variable deadband.

\subsection{Variable Threshold Alarm Systems with Variable deadband}

In this approach, use of variable threshold and deadband are merged to improve reduction of false and missed alarm probabilities. As mentioned in method 2, deadband is assumed as a percentage of alarm limits. Therefore, because of varying threshold, deadband should vary too.

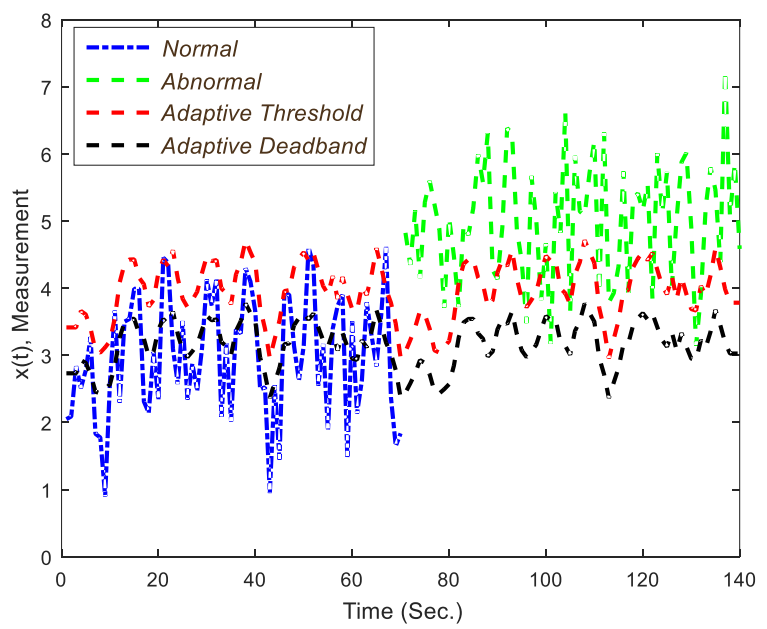

Fig. 5. Generated signal of example 1 with adaptive threshold and deadband

As seen in Fig. 5, variable deadband along with variable threshold is applied on the signal. Note that data of variable threshold and variable deadband have mean and variance, so for these data, pdf are considered. 


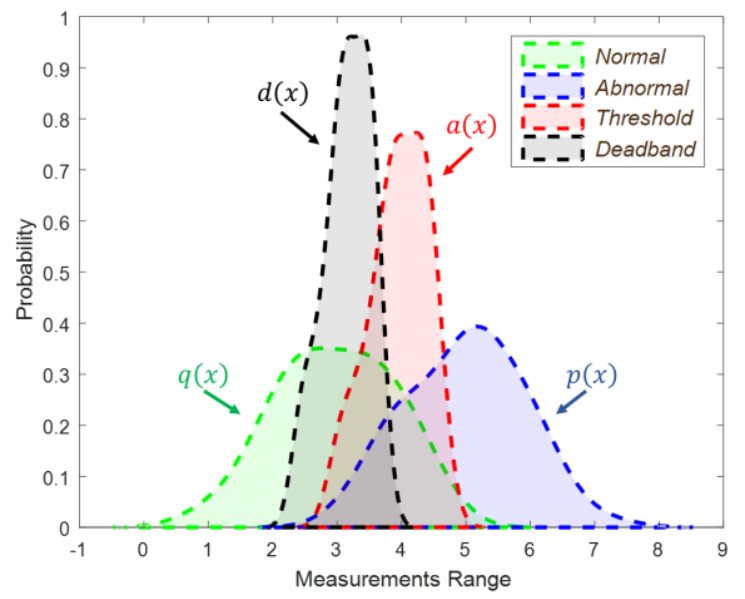

Fig. 6. The estimated probability density function of variable threshold, deadband, normal and abnormal signals.

In this method, in comparison with other methods, rate of false and missed alarm can be simultaneously reduced. This feature is the benefit of this method against other methods. Table 1 confirms this issue explicitly.

TABLE 1

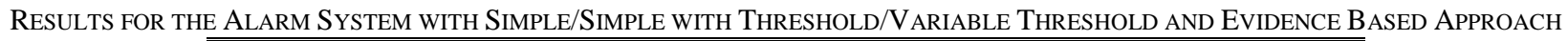

\begin{tabular}{ccccc}
\hline \hline $\begin{array}{c}\text { Performance } \\
\text { Ind./Method }\end{array}$ & $\begin{array}{c}\text { Simple } \\
\text { Threshold }\end{array}$ & $\begin{array}{c}\text { Variable } \\
\text { Threshold }\end{array}$ & $\begin{array}{c}\text { Simple Threshold } \\
\text { and deadband }\end{array}$ & $\begin{array}{c}\text { Variable } \\
\text { Threshold and } \\
\text { deadband }\end{array}$ \\
\hline \hline MAR & 0.2036 & 0.1836 & 0.0452 & 0.0448 \\
FAR & 0.2668 & 0.2009 & 0.2668 & 0.2009 \\
\hline \hline
\end{tabular}

\section{METHODOLOGY}

It is evident that in normal condition, when the value of $i^{\text {th }}$ measurement becomes larger than the threshold value ( $\mathrm{x}_{\mathrm{tp}}$ in simple threshold or related value of VTAS), the alarm is raised, and it is known as false alarm. Similarly, in an abnormal condition, when the value of $i^{\text {th }}$ measurement becomes smaller than the threshold value, the alarm returns to normal, and it is known as missed alarm. In other words, in normal condition, false alarm happened when $X_{Q}>X_{t p}$ and in the abnormal condition, missed alarm occurred when $X_{t p}>X_{P}$ in which $X_{Q}$ denotes measurements in normal condition, $X_{P}$ stands for measurement in an abnormal condition and $X_{t p}$ is the threshold value. To calculate the probability of false and missed alarm, a systematic method was needed.

In reliability engineering area, dynamic fault tree [37] is one the popular techniques which is helpful for systematic modeling and illustration of probabilistic and stochastic phenomena. In dynamic fault trees, a Priority-AND gate is used for sequential failure behavior modeling. If two events $\mathrm{A}$ and $\mathrm{B}$ are inputs to a PAND gate, then when the event A occurs before event B occurs, the output will be true $\left(t_{A}<t_{B}\right)$. This scenario in dynamic fault tree is depicted in Fig. 7-(a). In the literature related to dynamic fault tree analysis, several solutions such as [38, 39, 40, 41] have been provided for probabilistic evaluation of the PAND gate, given the occurrence rate or probability of the input events of the gate. In this paper, we utilize the behavior of the PAND gate for MAR and FAR assessments. In our methodology, if the PAND gate is used in $\mathrm{x}$ domain instead of time domain, we have a systematic and probabilistic gate in which inputs are CDF of normal measurements $(\mathrm{Q})$, abnormal measurements $(\mathrm{P})$ and variable threshold $(\mathrm{A})$. To achieve FAR, $X_{Q}>X_{t p}$ is a subject in normal condition and can be shown in Fig. 7-(b). In the same way, to obtain MAR, $X_{t p}>X_{P}$ is a subject in an abnormal condition and can be delineated as Fig. 7-(c). By the use of PAND gate, a systematic method for MAR and FAR calculation is available. As mentioned earlier, it is assumed that significant rise in measurements is a sign of abnormal behavior. On the contrary, if significant fall is measurements is considered as abnormal behavior, then the inputs of PAND gate in both MAR and FAR model must be switched. In this scenario, for the FAR model, "input A" must be defined as the first input and "input Q" as the second input of the gate respectively. For MAR model, "input P" must be set as the first input and "input A" as the second input of the gate, respectively. 


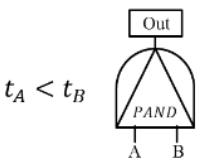

(a)

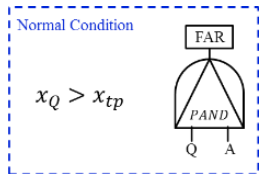

(b)

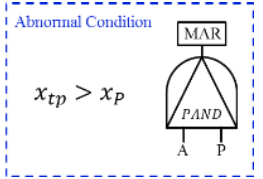

(c)

Fig. 7. (a) Illustration of PAND gate, (b) illustration of PAND gate for FAR calculation, (c) illustration of PAND gate for MAR calculation

With the same background, for VTAS with deadband, the FAR model can be depicted as Fig. 8-(a) and MAR model can be delineated as Fig. 8-(b). In these models, it is assumed that the alarm will be raised with raising faults. Thus, for falling faults these models must be rebuilt as explained before.

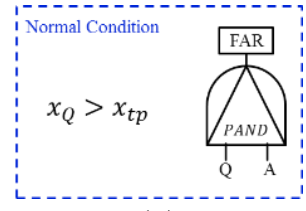

(a)

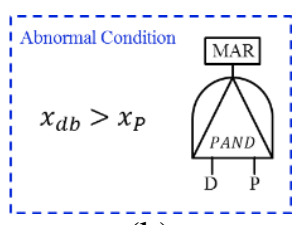

(b)

Fig. 8. VTAS with deadband, (a) illustration of PAND gate for FAR calculation, (b) illustration of PAND gate for MAR calculation

Solutions to above-mentioned gates will be introduced in the following in a logical way through the semi-Markov process and the numerical way through Trapezoidal integration. Fig. 9 illustrates the flowchart of the proposed method. This flowchart has two main loop for a specific type of fault; I) selecting sensors and their signals one by one II) optimizing the adaptive threshold for each sensor based on the KPIs evaluated through SMP. After completing both loops, the sensors with better KPIs will be selected and prioritized for that specific type of fault in the system that compressor fault, blade failure, or etc.

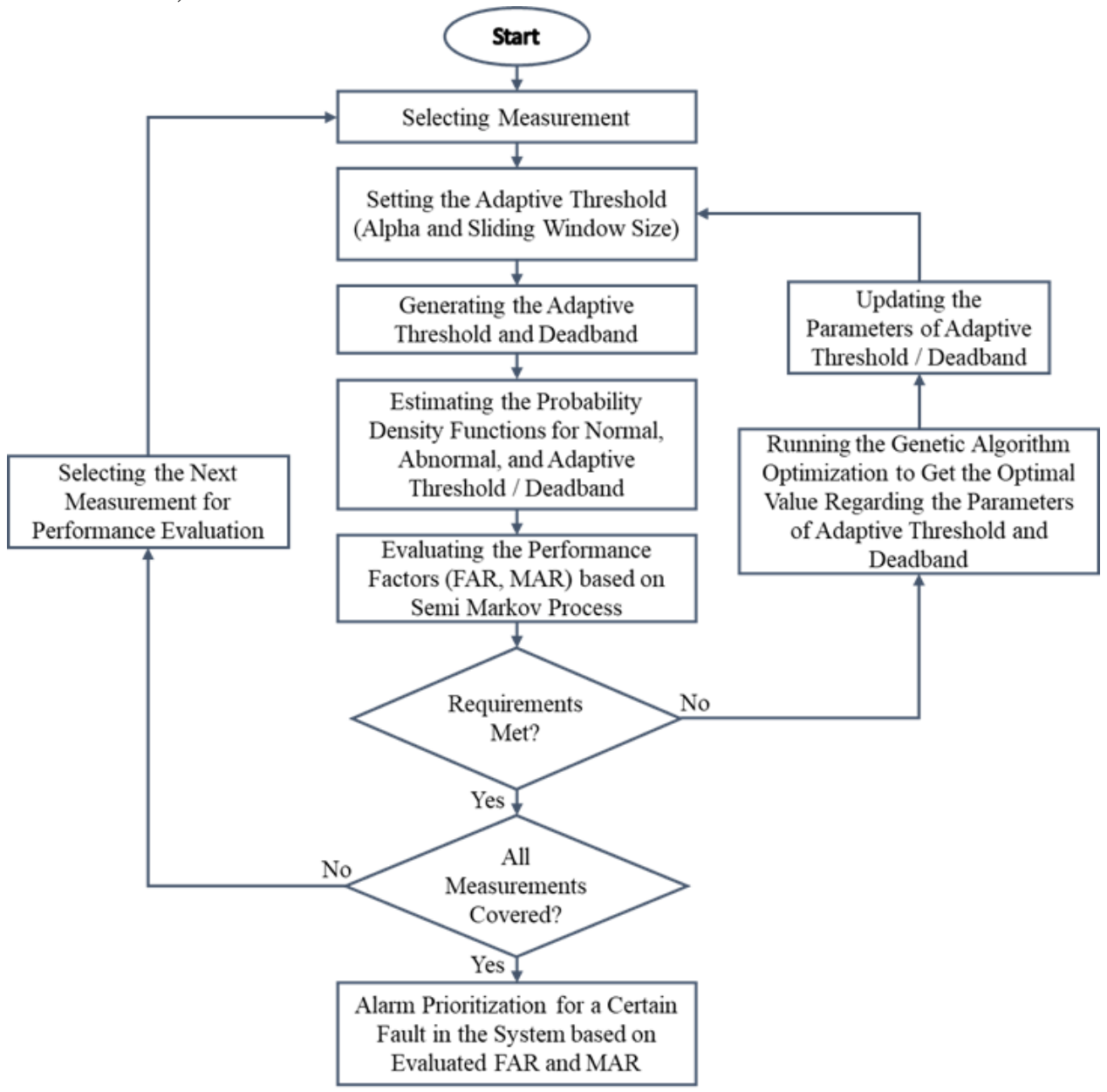

Fig. 9. Flow Chart of the proposed method 


\subsection{Assumptions}

Generally speaking, the industrial plants' alarm system deeply depends on different factors such as environmental effects, imperfection and operation modes that make the task of alarm system design challenging. In this paper, the following assumptions are made to increase the applicability of the proposed approach to a real industrial use.

- Measurements in this paper are assumed Independent and Identically Distributed (IID).

- The calculations will be provided for univariate VTAS, and multivariate performance evaluation remains for future research.

- The proposed method can model both type of falling and raising faults and their related alarm as mentioned before. However, in the rest of paper, it is assumed that in the alarm system only raising fault exists.

- It is assumed that in the design procedure of each VTAS, normal and abnormal measurements are classified by an expert at the beginning. Note that there are some unsupervised classification methods such as Fuzzy C-means [20] and one mean-change point detection [7] which can be applied to unlabeled data.

\subsection{SMP Analytical Solution}

In the literature, different types of solutions have been addressed, and all of them can be used for the assessment of MAR and FAR in VTAS. In this section, an analytical solution of SMP will be studied and then this theorem calculates the parametric probability of missed alarm and probability of false alarm through PAND gate. The SMP can be modeled by different notations [42]. This paper uses tuple (trio) ( $\mathrm{p}, \mathrm{P}, \mathrm{F}(\mathrm{x})$ ), where: $\mathrm{p}$ is vector of initial distribution, $\mathrm{P}$ is matrix of conditional transition probabilities and $\mathrm{F}(\mathrm{x})$ describes matrix of distribution functions of measurements in state $i$, when $j^{\text {th }}$ state is next; Considering $X_{i}, \forall i=0,1,2, \ldots$ as a random variable, the homogeneous SMP X is determined by a vector of initial state probabilities $p(0)=\left[P\left\{X_{0}=i\right\}\right]=[1,0, \ldots, 0]$, and the conditional transition probability matrix $P(x)=\left[P_{i j}(x)\right]$. where $P(x)$ is computed by (13).

$$
P_{i j}(x)=P\{X(x)=j \mid X(0)=i\} \quad i, j \in \text { States }
$$

The conditional probabilities' matrix $\left(P_{i j}(x)\right)$ is satisfied by Kolmogorov-Feller's equation in (14) [43].

$$
P_{i j}(x)=\delta_{i j}\left[1-G_{i}(x)\right]+\sum_{K \in S} \int_{0}^{x} P_{k j}(t-y) d Q_{i k}(y)
$$

where $\delta_{i j}=1$ if $i=j$ and $\delta_{i j}=0$ otherwise, $G_{i}$ is the distribution in state $i$ described by (15) [44, 45] and $Q_{i j}(x)$ represent the kernel matrix by (16). Note that the kernel matrix has a Markov renewal theorem in the background [46].

$$
G_{i}(x)=P\left\{S_{1} \leq x \mid X_{0}=i\right\}=\sum_{j=1}^{i} Q_{i j}(x)
$$

where $S_{i}, i=0,1,2, \ldots$ is the states of the system at different $\mathrm{x}$.

$$
Q_{i j}(x)=P\left\{X_{1}=j, S_{1} \leq x \mid X_{0}=i\right\}
$$

A solution to (16) can be found by applying Laplace Stieltjes Transformation (LST) in (17) [47]. There is a set of Voltera equations in (14) which is a Markov renewal equation [44]. Note that for non-exponential failure distributions such as Weibull and Gamma, some approximation is needed (Refer to [48, 42, 49]).

$$
\tilde{p}_{i j}(s)=\delta_{i j}\left[1-\tilde{g}_{i}(s)\right]+\sum_{K \in S} \tilde{q}_{i k}(s) \tilde{p}_{k j}(s)
$$

Equation (17) in the matrix form can be rewritten as follows:

$$
\tilde{p}(s)=[I-\tilde{g}(s)]+\tilde{q}(s) \tilde{p}(s)
$$

Hence, it can be rewritten as (19).

$$
\tilde{p}(s)=[1-\tilde{q}(s)]^{-1}(I-\tilde{g}(s))
$$

In (19), the inverse of $1-\tilde{q}(s)$ can be replaced by the summation of powers of $\tilde{q}(s)$. The resulted equation which is useful for singular kernel matrix will be as (20).

$$
\tilde{p}(s)=\left(\sum_{n=0}^{\infty} \tilde{q}(s)^{n}\right)(I-\tilde{g}(s))
$$


Having solved (20) by taking the inverse LST of $\tilde{p}(s)$, the unconditional state probabilities in $\mathrm{x}$ domain are determined as follows:

$$
P(x)=P(0) P(x)
$$

Finally, the probability of missed alarm or false alarm can be achieved by summing up the transient probability of some states which is discussed more in next sub-section.

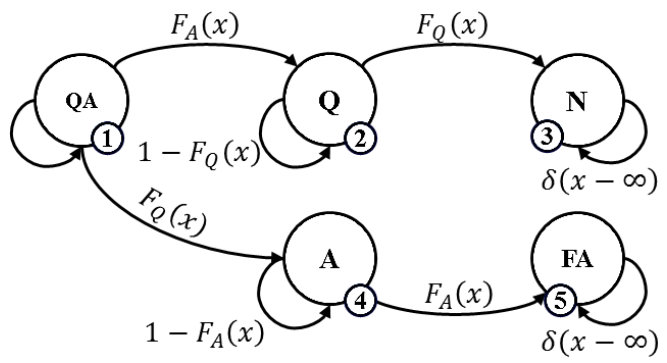

Fig. 10. Semi-Markov model of PAND gate for FAR

\subsection{MAR and FAR Evaluation of a Measurement with Exponential pdf}

To ease the demonstration of the proposed method, this paper considered exponential pdf for both abnormal and normal measurements. Markov model of PAND gate has been presented in the literature [50, 51]. Fig. 9 illustrates the semi-Markov model of the PAND gate with any types of the distribution function. In this model, $F_{A}(x)$ is Cumulative Distribution Function (CDF) of the second input of the PAND gate (CDF of variable threshold) and $F_{Q}(x)$ is CDF of the first input of the PAND gate (CDF of measurement), respectively. States are numbered from 1 to 5 from top to bottom and left to right. This model can be solved by SMP theorem described in the previous sub-section.

The kernel matrix of Fig. 9 can be written in the form of (22) and distribution matrix of each state is in the form of (23).

$$
\begin{aligned}
& Q(x)= {\left[\begin{array}{ccccc}
0 & Q_{1,2} & Q_{1,4} & 0 & 0 \\
0 & 0 & 0 & Q_{2,3} & 0 \\
0 & 0 & 0 & 0 & Q_{4,5} \\
0 & 0 & 0 & 0 & 0 \\
0 & 0 & 0 & 0 & 0
\end{array}\right] } \\
& G(x)= \operatorname{diag}\left(1-\left(1-F_{A}(x)\right)\left(1-F_{Q}(x)\right), F_{A}(x),\right. \\
&\left.F_{Q}(x), \delta(x-\infty), \delta(x-\infty)\right)
\end{aligned}
$$

In matrix (22), $Q_{1,2}$ can be defined as (24), for further explanation of this definition refer to [44].

$$
\begin{aligned}
Q_{1,2} & =\operatorname{Pr}\left\{\mathrm{X}_{1}=2, S_{1} \leq x \mid X_{0}=1\right\} \\
& =\operatorname{Pr}\left\{\mathrm{L}_{Q} \leq x \wedge L_{A} \leq L_{Q}\right\}=\int_{0}^{x} 1-F_{A}(y) d F_{Q}(y)
\end{aligned}
$$

For exponential CDF, in the case of A and Q, (24) can be written as:

$$
\begin{aligned}
Q_{1,2} & =\int_{0}^{x}\left[1-\left(1-e^{-\lambda_{A} \tau}\right)\right] d\left\{1-e^{-\lambda_{Q} \tau}\right\} \\
& =\int_{0}^{x} e^{-\left(\lambda_{A}+\lambda_{Q}\right) \tau} \lambda_{Q} d \tau=\frac{\lambda_{Q}\left[1-e^{-\left(\lambda_{A}+\lambda_{Q}\right) x}\right]}{\lambda_{A}+\lambda_{Q}}
\end{aligned}
$$

Similarly, $Q_{1,4}$ can be defined as (26). For exponential CDF of both inputs, (26) can be written as (27).

$Q_{1,4}=\operatorname{Pr}\left\{\mathrm{X}_{1}=4, S_{1} \leq x \mid X_{0}=1\right\}$

$=\operatorname{Pr}\left\{\mathrm{L}_{A} \leq x \wedge L_{Q} \leq L_{A}\right\}$ 


$$
\begin{aligned}
Q_{1,4} & =\int_{0}^{x}\left[1-\left(1-e^{-\lambda_{Q} \tau}\right)\right] d\left\{1-e^{-\lambda_{A} \tau}\right\} \\
& =\int_{0}^{x} e^{-\left(\lambda_{A}+\lambda_{Q}\right) \tau} \lambda_{A} d \tau=\frac{\lambda_{A}\left[1-e^{-\left(\lambda_{A}+\lambda_{Q}\right) x}\right]}{\lambda_{A}+\lambda_{Q}}
\end{aligned}
$$

$Q_{2,3}$ is the probability of occurrence of input A and $Q_{4,5}$ is the probability of occurrence of input Q. $Q_{2,3}$ and $Q_{4,5}$ are defined by (28) and (29).

$Q_{2,3}=\operatorname{Pr}\left\{\mathrm{X}_{1}=3, S_{1} \leq x \mid X_{0}=2\right\}$

$Q_{4,5}=\operatorname{Pr}\left\{\mathrm{X}_{1}=5, S_{1} \leq x \mid X_{0}=4\right\}$

In exponential CDF form (28) and (29) are written by (30) and (31), respectively.

$Q_{2,3}==\int_{0}^{x} \lambda_{A} e^{-\lambda_{A} \tau} d \tau=1-e^{-\lambda_{A} x}$

$Q_{4,5}==\int_{0}^{x} \lambda_{Q} e^{-\lambda_{Q} \tau} d \tau=1-e^{-\lambda_{Q} x}$

The LST of kernel matrix is written as (32) in its general form.

$\tilde{q}(s)=\left[\begin{array}{ccccc}0 & L\left\{Q_{1,2}\right\} & L\left\{Q_{1,4}\right\} & 0 & 0 \\ 0 & 0 & 0 & f_{A}^{*}(s) & 0 \\ 0 & 0 & 0 & 0 & f_{Q}^{*}(s) \\ 0 & 0 & 0 & 0 & 0 \\ 0 & 0 & 0 & 0 & 0\end{array}\right]$

In general form, the LST of G matrix is written as (33). In exponential form, it is written as (34). Note that "diag" creates a diagonal matrix from each input vector.

$$
\begin{aligned}
& \tilde{g}(s)=\operatorname{diag}\left(L\left\{1-\left(\left(1-F_{A}(x)\right)\left(1-F_{Q}(x)\right)\right)\right\}, f_{A}^{*}(s), f_{Q}^{*}(s), 1,1\right) \\
& I-\tilde{g}(s)=\operatorname{diag}\left(\frac{s}{s+\lambda_{A}+\lambda_{Q}}, \frac{s}{s+\lambda_{A}}, \frac{s}{s+\lambda_{Q}}, 1,1\right)
\end{aligned}
$$

Similarly, (35) is LST of kernel matrix for exponential CDF.

$$
\tilde{q}(s)=\left[\begin{array}{ccccc}
0 & \frac{\lambda_{Q}}{s+\lambda_{A}+\lambda_{Q}} & \frac{\lambda_{A}}{s+\lambda_{A}+\lambda_{Q}} & 0 & 0 \\
0 & 0 & 0 & \frac{\lambda_{A}}{s+\lambda_{A}} & 0 \\
0 & 0 & 0 & 0 & \frac{\lambda_{Q}}{s+\lambda_{Q}} \\
0 & 0 & 0 & 0 & 0 \\
0 & 0 & 0 & 0 & 0
\end{array}\right]
$$

By using equations (19) or (20), the unconditional probability vector of Fig. 9 is compute as:

$\widehat{P}=\left[L^{-1}\left\{\left(1-f_{A}^{*}(s)\right)\left(1-f_{Q}^{*}(s)\right)\right\}\right.$,

$L^{-1}\left\{q_{1,2}(s)\left(1-f_{A}^{*}(s)\right)\right\}, L^{-1}\left\{q_{1,4}(s)\left(1-f_{Q}^{*}(s)\right)\right\}$

$\left.L^{-1}\left\{q_{1,2}(s)\left(f_{A}^{*}(s)\right)\right\}, \quad L^{-1}\left\{q_{1,4}(s)\left(f_{Q}^{*}(s)\right)\right\}\right]$

Finally, the probability of PAND gate output can be obtained from the probability of fail state (state 5 in Fig. 10) using (37).

$$
F A R=\widehat{P}_{5}(x)=L^{-1}\left\{q_{1,4}(s)\left(f_{Q}^{*}(s)\right)\right\}
$$

For example, the probability of a PAND gate output with exponential CDF can be extracted as (38). 


$$
\begin{aligned}
& F A R=L^{-1}\left\{\left(\frac{\lambda_{A}}{s+\lambda_{A}+\lambda_{Q}}\right)\left(\frac{\lambda_{Q}}{s+\lambda_{Q}}\right)\right\} \\
& F A R=1-\left(e^{-\lambda_{Q} x}+\frac{\lambda_{Q}\left(1-e^{-\left(\lambda_{A}+\lambda_{Q}\right) x}\right)}{\lambda_{A}+\lambda_{Q}}\right)
\end{aligned}
$$

Similarly, if the SMP model of MAR is drawn as Fig. 11, the probability of missed alarm or MAR will be obtained as (39).

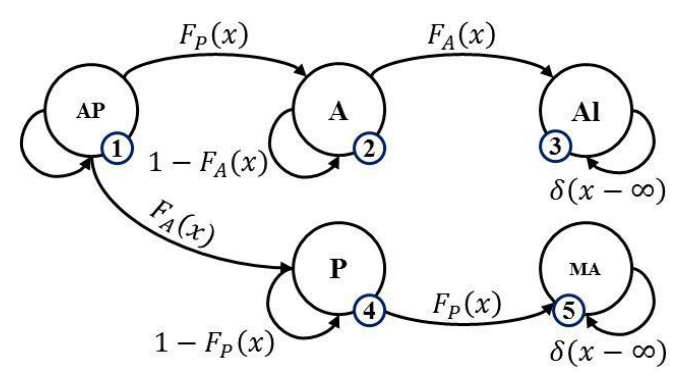

Fig. 11. Semi-Markov model of PAND gate for MAR

$$
\operatorname{MAR}=\widehat{P}_{5}(x)=L^{-1}\left\{q_{1,4}(s)\left(f_{P}^{*}(s)\right)\right\}
$$

When both normal and abnormal measurements obey exponential distribution, MAR will be (40).

$$
\begin{aligned}
& M A R=L^{-1}\left\{\left(\frac{\lambda_{A}}{s+\lambda_{A}+\lambda_{P}}\right)\left(\frac{\lambda_{P}}{s+\lambda_{P}}\right)\right\} \\
& M A R=1-\left(e^{-\lambda_{P} x}+\frac{\lambda_{P}\left(1-e^{-\left(\lambda_{A}+\lambda_{P}\right) x}\right)}{\lambda_{A}+\lambda_{P}}\right)
\end{aligned}
$$

\subsection{Validation through Monte Carlo Simulation}

The Monte Carlo Simulation with ten million repetition is used to validate the proposed Semi-Markov based approach. The example 1 is considered and in each iteration, false positive and negative values of generated confusion matrix has been stored in a vector. Having finished the simulation, the mean and variance values of false positive and false negative is calculated as the probability of false alarm and probability of missed alarm respectively. The comparison between Semi-Markov based solution and Monte Carlo Simulation is provided in Table 2.

TABLE 2

Monte Carlo Simulation in Comparison with Semi-Markov Based Solution

\begin{tabular}{cccc}
\hline \hline \multirow{2}{*}{ Performance Ind./Method } & \multicolumn{2}{c}{ Monte Carlo Simulation (Ten Million Repetition) } & \multirow{2}{*}{ Semi-Markov Based Solution } \\
\cline { 2 - 3 } & Mean Value & Variance Value & 0.15867 \\
\hline \hline Probability of Missed Alarm & 0.159856 & $1.34 \mathrm{e}-04$ & 0.15867 \\
Probability of False Alarm & 0.159852 & $1.34 \mathrm{e}-04$ & 04 \\
\hline \hline
\end{tabular}

$\square$ According to the above table the correctness of the Semi-Markov based approach has been validated.

\subsection{Numerical Solution}

In most industrial cases, there is no parametric pdf and CDF for measurements, and usually, they are obtained numerically. Equations (41) and (42) are provided from [52] for a numerical solution in which integrations are "Trapezoidal numerical integration".

$$
\begin{aligned}
& F A R=\int_{0}^{x 1} f_{A}(x) F_{Q}(x) d x \\
& M A R=\int_{0}^{x 2} f_{P}(x) F_{A}(x) d x
\end{aligned}
$$

Similarly, in the case of VTAS with Deadband, (41) and (42) can be rewritten as (43) and (44). 


$$
\begin{aligned}
& F A R=\int_{0}^{x 1} f_{A}(x) F_{Q}(x) d x \\
& M A R=\int_{0}^{x 2} f_{P}(x) F_{D}(x) d x
\end{aligned}
$$

where $F_{D}(x)$ is the CDF of variable deadband. In the equation 41 to 44 , numerical probability distribution and density vector should be used for $\mathrm{F}$ and $\mathrm{f}$ respectively.

\subsection{Optimization through Genetic Algorithm}

The multi-objective and evolutionary algorithms can be considered for designing the optimal alarm system. Genetic Algorithm (GA) is one of the well-known meta-heuristics algorithms to find solutions to optimization problems. The first step in GA is to generate an initial population of random solutions. The next is step is the selection operation, in which two or more solutions are selected from the current population using a selection function. Afterwards, crossover operation is performed on these solutions to generate new solutions. Then the new solutions are mutated with a given mutation probability.

In this approach, the input variables (number of sample alarm in both normal and abnormal measurements $(n, m)$, the width of sample window and alpha in VTAS) are assumed as genotype and the phenotype will be the probability of false alarm, probability of missed alarm and average alarm detection. The distributions of inputs obey uniform function where $n$ and $m$ have the low value of one and high value of twenty. The alpha parameter has the range of twenty to thirty. Moreover, window length has the range of twenty to hundred. Top genes with lowest cost in each generation is chosen as the input of crossover function to generate next population. Meanwhile, the mutation function generate new random genes and add them to next population. This procedure will be repeated for a certain number of iterations to achieve optimal value (lowest cost) [30].

In the optimization, forty population is considered and in each generation; the rate of mutation is considered thirty five percent, the rate of single point crossover is considered fifty percent and the rate of recombination is considered fifteen percent.

Alarm system design, alarm delay and AAD depends on some alarm samples $(n-m)$ and increasing $n$ or $m$ leads to increase in the AAD value. Also, regardless of the effect of $\alpha$ and $w$ on MAR and FAR, in most cases, increasing the number of alarm samples $n, m$ leads to decreasing the MAR and FAR values. To make a trade-off between optimization of AAD, MAR, and FAR, the weighted-sum cost function can be used as (45) [15]. Note that the analytic expressions of MAR, FAR and AAD related to $\mathrm{n}$ and $\mathrm{m}$ have been derived from [53].

$$
J(\alpha, w, n, m)=\omega_{1} \frac{F A R}{R F A R}+\omega_{2} \frac{M A R}{R M A R}+\omega_{3} \frac{A A D}{R A A D}
$$

where RFAR, RMAR, and RAAD are the requirements of FAR, MAR, and AAD, respectively. Note that, because of various delay in $\mathrm{n}-\mathrm{m}$ sample alarm delay, AAD is divided into two parts $A A D_{n}$ and $A A D_{m}$ for $\mathrm{n}$ and $\mathrm{m}$ sample of delay, respectively. Depending on the practice situation, the importance of each FAR, MAR and AAD can be weighted via $\omega_{1}, \omega_{2}, \omega_{3}$ and $\omega_{4}$, respectively. The introduced weight can be chosen differently depending on the application. For example, if in the hypothetical industrial system, delay reduction is the most important issue and MAR has priority over FAR, the weights of cost function $J(\alpha, w, m, n)$ should be considered as $\omega_{1}<\omega_{2}<\omega_{3}=\omega_{4}$. As a result of GA optimization, the optimal value of "number of alarm samples (n, m)", "alpha coefficient of adaptive threshold" and "window size of adaptive threshold" can be achieved. In other words, the aim of optimization problem can be written as follows:

$$
(\alpha, w, n, m)=\arg \min J(\alpha, w, n, m)
$$

In the following, the effectiveness of the proposed penalty scenario and GA optimization is illustrated with some industrial case study measurements.

\section{NUMERICAL RESULTS}

In this section, three numerical examples are provided to illustrate the capabilities of Semi-Markov based solution and compare the effectiveness of proposed method with other published research. 


\subsection{Example 2: Measurements with Weibull Distribution}

In previous sections, an example of measurements with piecewise white Gaussian random distribution was investigated, and this example is given to show the effectiveness of the proposed method in non-Gaussian random distribution. For this reason, the Weibull distribution is considered with the following expression. It is assumed that the length of generated data is 200 in which measurements from 0 to 100 obey $F_{\text {nomal }}$ and measurements from 101 to 200 follow $F_{\text {abnormal }}$.

$$
\begin{aligned}
& F_{\text {normal }}(x)=1-e^{-\left(\frac{x}{A}\right)^{B}}, A=1, B=1.3 \\
& F_{\text {abnormal }}(x)=1-e^{-\left(\frac{x}{A}\right)^{B}}, A=5, B=2
\end{aligned}
$$

The pdf curves of both normal and abnormal hypothetical measurements are illustrated in Fig. 12.

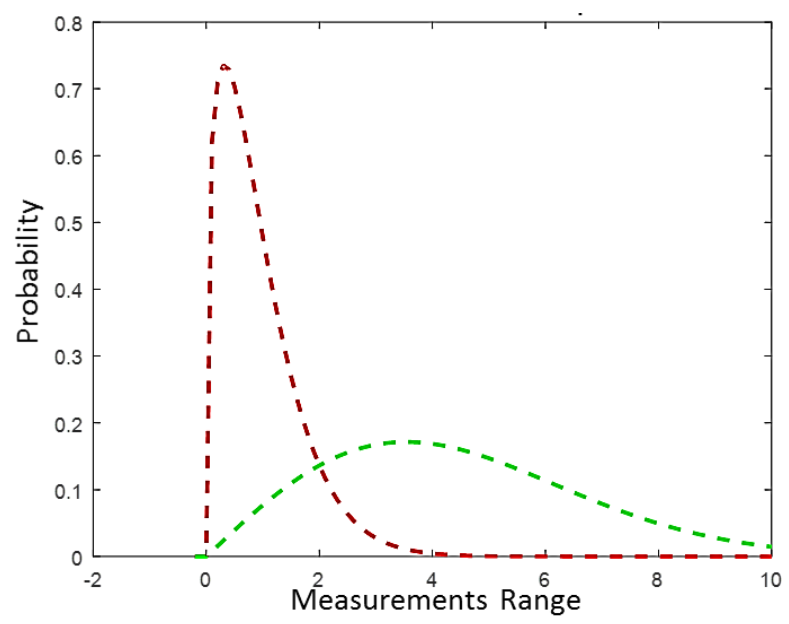

Fig. 12. Probability density functions of both normal (red) and abnormal (green) measurements (Weibull distribution)

Table 3 shows the computed MAR and FAR through Monte Carlo Simulation, the proposed method, and simple Markov solution respectively. As can be seen in this table, results of the proposed method and Monte Carlo simulation are same. However, there are two ways to use simple Markov solution; I) computed mean and variance of normal and abnormal measurements and using analytical normal distribution expression or II) using numerical pdf estimation. The results of simple Markov-based solutions are inexact for this example because of the non-Gaussian behavior of measurements.

TABLE 3

Comparison between the Proposed Solution, Monte Carlo Results, and Simple Markov Solution

\begin{tabular}{ccccccc}
\hline \hline \multirow{2}{*}{ Performance Ind./Method } & \multicolumn{2}{c}{ Monte Carlo (1e06 Iteration) } & Proposed Solution & $\begin{array}{c}\text { Markov } \\
\text { (Using Normal } \\
\text { pdf) }\end{array}$ & $\begin{array}{c}\text { Markov (Using } \\
\text { Estimated pdf) }\end{array}$ \\
\cline { 2 - 3 } & Mean & Variance & & 0.0852 & 0.1371 & 0.1070 \\
FAR & 0.0852 & $7.8019 \mathrm{e}-4$ & 0.1478 & 0.1371 & 0.1455 \\
\hline \hline
\end{tabular}

\subsection{Example 3: Measurements with Gaussian Distribution}

This example is taken from [18] in which the hypothetically generated measurements $x(t)$ is as a "piecewise white Gaussian random variable" with imprecise mean and variance as (48). The sample rate of random number generation is one second and $x(t)$ is shown in Fig. 13. 


$$
\left\{\begin{array}{l}
x(t)=N\left(\mu_{1}, \sigma_{1}^{2}\right)\left\{\begin{array}{l}
\mu_{1}=U(0.2,0.3) \\
\sigma_{1}=U(1.5,1.6)
\end{array} \quad t<2000\right. \\
x(t)=N\left(\mu_{2}, \sigma_{2}^{2}\right)\left\{\begin{array}{l}
\mu_{2}=U(1.2,1.5) \\
\sigma_{2}=U(1.5,1.6)
\end{array} \quad t \geq 2000\right.
\end{array}\right.
$$

where $\mathrm{U}$ denotes random variables with uniform distributions.

In this example, the static distributions of hypothetical measurements are partially known and imprecise because $x(t)$ has the mixture of epistemic and aleatory uncertainties. As can be seen in Fig. 13, this is a challenging example for simple threshold decision making. However, by the use of Exponential Weighted Moving Average (EWMA) filter with one sample delay and weight coefficient of 0.05 [1], Fig. 14 can be obtained from Fig. 13.

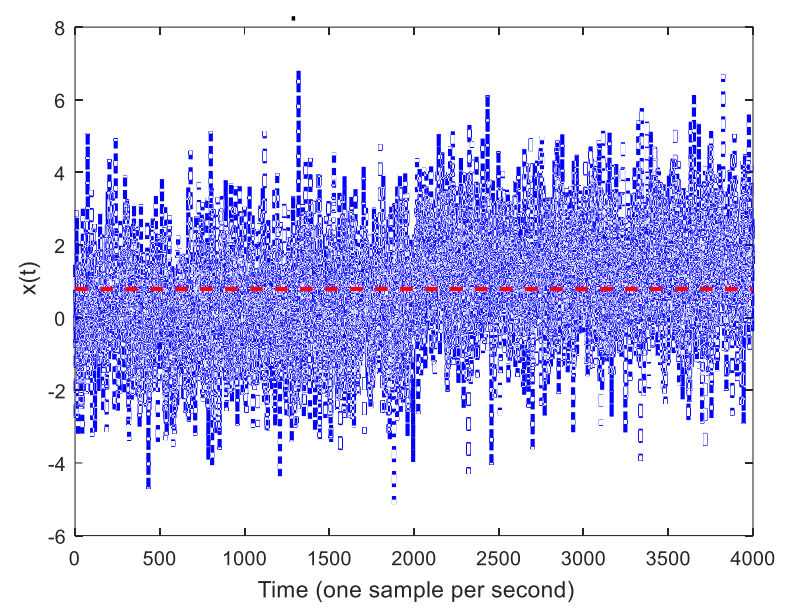

Fig. 13. Random number generated based on (48) [18]

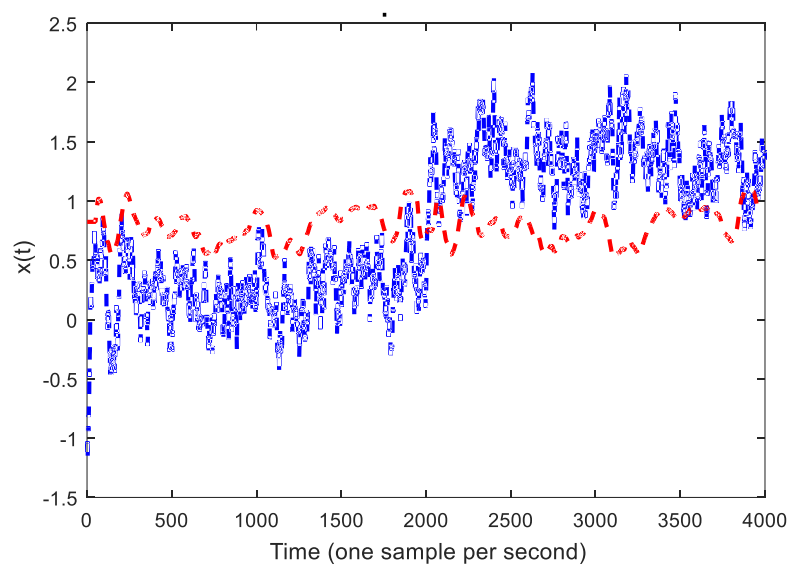

Fig. 14. Random number generated based on (48) after using EWMA filter with adaptive threshold $($ alpha $=0.5$, window size $=100)$

It is clear that the use of EWMA filter eases the decision-making procedure and improve both MAR and FAR. It is evident that this paper follows a systematic way for MAR and FAR computation. Therefore, in such kind of examples where the use of filtering is needed, it is suggested to use additional theorem like [14] besides our proposed method.

Table 4 provides a comparison result in performance indices (MAR and FAR) between variable threshold and other existing methods such as simple threshold and EWMA alarm system [1], evidence-based alarm system [18], and 3order moving average filter (3OMAF) [18], 3-sample alarm delay timer (3SADT) [7]. According to these results, the evidence-based alarm system has better performance indices than other methods. However, the VTAS has a better result than the simple threshold alarm system. 
TABLE 4

Comparative Results of Performance Indices between Variable Threshold and Evidence Based Alarm System

\begin{tabular}{cccccccc}
\hline \hline $\begin{array}{c}\text { Performance } \\
\text { Ind./Method }\end{array}$ & ST [1] & VT & EWMA [1] & $\begin{array}{c}\text { VT } \\
\text { (+ EWMA } \\
\text { filter) }\end{array}$ & $\begin{array}{c}\text { Evidence- } \\
\text { Based Alarm } \\
\text { System [18] }\end{array}$ & 3OMAF [18] & 3SADT [7] \\
\hline \hline MAR & 0.3519 & 0.3118 & 0.0437 & 0.0518 & 0.0582 & 0.2470 \\
FAR & 0.3806 & 0.2860 & 0.0447 & 0.0241 & 0.0429 & 0.2926 \\
\hline \hline
\end{tabular}

\subsection{Industrial Implementation: V94-2 Gas Turbine}

Fig. 15 depicts the schematic of a typical gas turbine which is a well-known and widely used for electricity production in the industrial power plants. In this turbine air from air intake enters the system and then compressed by the compressor unit to reach a certain level of high-pressure air. Having injected the high-pressure air into the combustor, it will be mixed with fuel that generates a flow of high-pressure combustion gas. This flow causes the coupled shaft rotation and consequently electricity generation through an electrical generator. The heated gases can exit from the exhaust to be wasted or used in the second or third rounds [23]. As an experimental research work, the proposed VTAS has been implemented for a V94-2 gas turbine [54, 23] of Fars power plant, Iran. In this turbine, alarm system collaborates with measurements of 108 sensors, which is embedded into the system. Measurements of vibration sensor as a challenging data is considered to show the effectiveness of the proposed method.

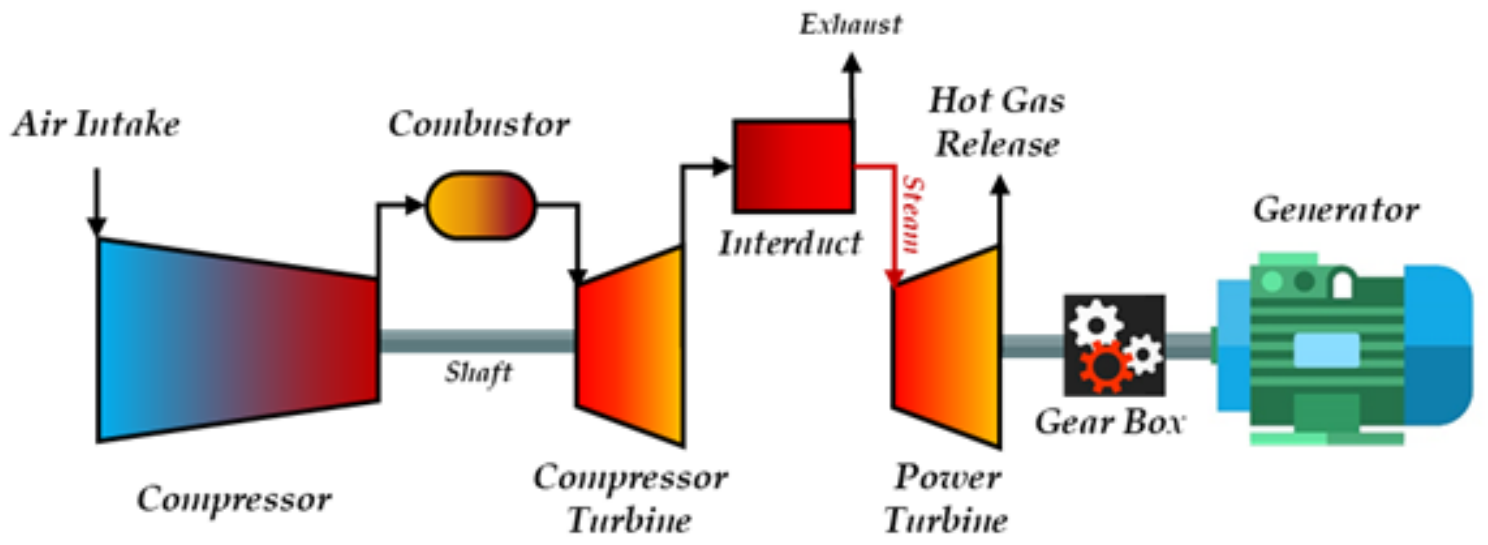

Fig. 15. Simple Gas Turbine Diagram

Fig. 16 illustrates the output signal of vibration sensor in which blue color of the curve shows the abnormal condition (before systems overhaul) and green color of the curve depicts the normal condition (after systems overhaul). In this figure, designed adaptive threshold is delineated with red color. Note that the abnormal condition of measurements is related to the compressor fault of the mentioned turbine. The unit for the vibration signal is millimeter per second $(\mathrm{mm} / \mathrm{s})$ and the range of variation in normal condition would be from 0.75 to $0.85 \mathrm{~mm} / \mathrm{s}$. It is assumed that there is no very-short-duration intermittent type fault in the system. Thus, the observed spikes in normal signal (Green) are not considered as abnormal event or condition. They can be an intermittent fault, a high amplitude noise or a disturbance. However, even if those spikes are abnormal, based on our assumption we have to ignore them. 


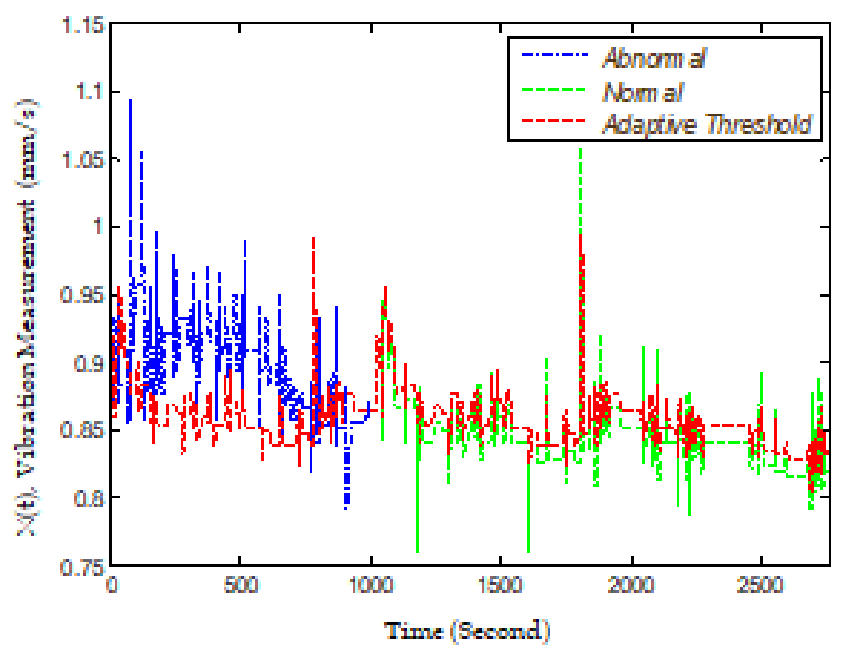

Fig. 16. Measurements of vibration sensor of V94-2 gas turbine by applying adaptive threshold (alpha=20)

In Fig. 17, probability density function of measurements in normal/abnormal conditions and designed adaptive threshold are illustrated by green, blue and red color respectively. This figure shows the estimated probability density function of variable/adaptive threshold follows similar pattern as the probability density function of normal data as can be expected.

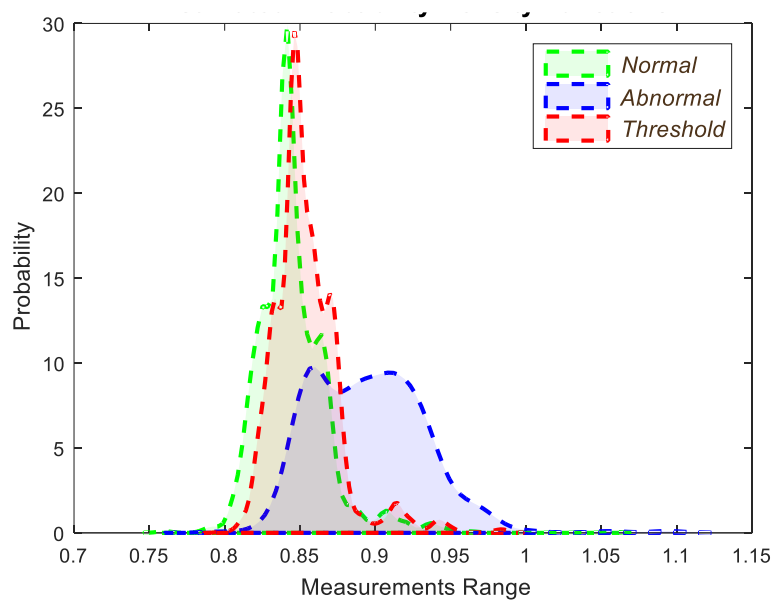

Fig. 17. Probability density function of vibration sensor of V94-2 gas turbine in normal and abnormal conditions

Table 5 depicts comparative results of performance evaluation of variable threshold and simple threshold alarm systems. As can be seen the rate of missed alarm decreases by the use of simple threshold with deadband. Moreover, the rate of false alarm increases in the variable threshold system. However, variable threshold system can reduce MAR better than the others.

TABLE 5

Comparative Results of Performance Indices between Variable Threshold and Simple Static Threshold

\begin{tabular}{cccc}
\hline \hline $\begin{array}{c}\text { PERFORMANCE } \\
\text { IND./METHOD }\end{array}$ & $\begin{array}{c}\text { SiMPLE } \\
\text { THRESHOLd }\end{array}$ & $\begin{array}{c}\text { VARIABLE } \\
\text { THRESHOLd }\end{array}$ & $\begin{array}{c}\text { SiMPLE } \\
\text { THRESHOLD (WITH } \\
\text { DEADBAND) }\end{array}$ \\
\hline \hline MAR & 0.460713 & 0.207569 & 0.364654 \\
FAR & 0.192783 & 0.289527 & 0.228910 \\
\hline \hline
\end{tabular}

According to GA optimization section, different requirements lead to various results of optimization. In other words, based on our priority and determined cost function coefficients, different values for VTAS will be obtained. Table 6 is given to illustrate different situations in GA and its results. For example, in the first result, it is assumed that there is no sample delay, and VTAS parameters ( $\boldsymbol{\alpha}$ and width of window) is obtained with lowest possible performance indices. As seen in Table 6, when the use of sample delay is allowed, the value of AAD will be increased, and both MAR and FAR will be decreased as expected. 
TABLE 6

OPTIMIZATION OF VTAS THROUGH GENETIC ALGORITHM

\begin{tabular}{|c|c|c|c|c|c|c|}
\hline \multicolumn{3}{|c|}{ Cost function's weights in GA } & \multirow{2}{*}{$\begin{array}{c}\begin{array}{c}\text { Parameters of Alarm } \\
\text { system }\end{array} \\
(n, m, \alpha, w)\end{array}$} & \multicolumn{3}{|c|}{ Performance Indices } \\
\hline & & & & MAR & FAR & AAD \\
\hline $\begin{array}{c}\omega_{3} \\
100 \\
\text { RAAD } \\
1\end{array}$ & $\begin{array}{c}\omega_{2} \\
0.1 \\
\text { RFAR } \\
1 \mathrm{e}-03 \\
\end{array}$ & $\begin{array}{c}\omega_{1} \\
0.1 \\
\text { RMAR } \\
1 \mathrm{e}-03 \\
\end{array}$ & $(2,3,25.039,82)$ & 0.0556 & 0.1681 & 2.8742 \\
\hline $\begin{array}{c}\omega_{3} \\
100 \\
\text { RAAD } \\
1\end{array}$ & $\begin{array}{c}\omega_{2} \\
0.5 \\
\text { RFAR } \\
1 \mathrm{e}-03\end{array}$ & $\begin{array}{c}\omega_{1} \\
0.1 \\
\text { RMAR } \\
1 \mathrm{e}-03\end{array}$ & $(2,3,25.6803,83)$ & 0.0596 & 0.1625 & 2.897 \\
\hline
\end{tabular}

It is obvious that presence of different faults in a system has different signs and effects on its measurements. In other words, the presence of a fault has a significant effect on some measures and a small effect on the other ones. So alarm prioritization could be necessary for these systems [55]. In the aforementioned gas turbine, for compressor fault, a prioritization algorithm has been used in which 108 sensors' measurements of gas turbine is sorted based on their MAR, FAR, and the average of both MAR and FAR.
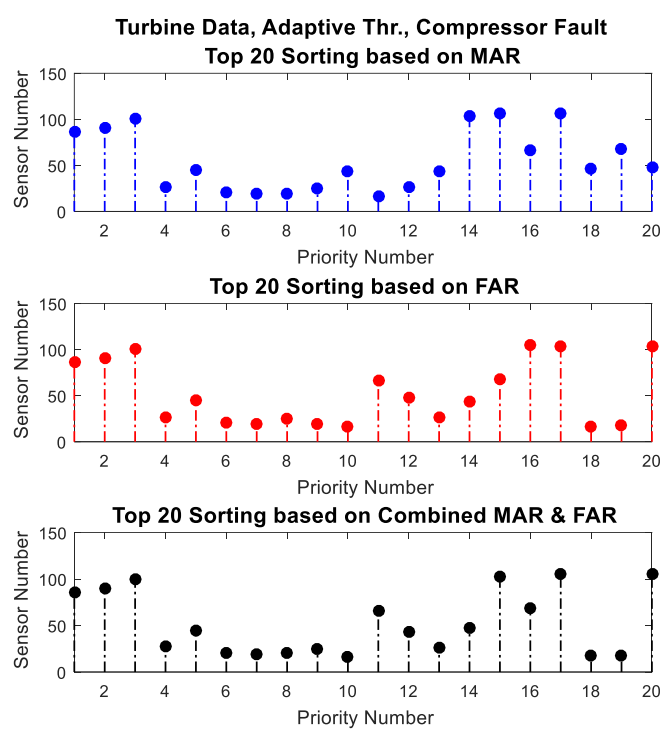

Fig. 18. Sensor sorting based on MAR, FAR and combined MAR and FAR for compressor fault in V94-2 Gas Turbine

Fig. 18 shows the top 20 sorted sensors based on MAR, FAR and combined MAR and FAR, respectively. The horizontal axes of these charts stand for priority number, and the vertical axes of them show the sensor number. As an example, in this figure, sensor 86 has top priority based on its MAR and FAR. It means that this sensor has the lowest correlation between normal and abnormal measurements, and achieved the highest priority in compressor fault detection or alarm. Note that, in this prioritization, the adaptive threshold is considered, and similarly these results can be easily generated for other thresholding method and other faults of the turbine.

\section{CAPABILITIES AND WEAK POINTS OF THE INTRODUCED SOLUTION}

In this section, the main contributions of the proposed method are discussed, and also the weak points of the solution with guidelines to overcome are suggested.

\subsection{Proposed Method and its Capabilities}

The proposed method has the following capabilities: 
1) In this paper, adaptive threshold with and without adaptive deadband has been introduced with a unique methodology for performance assessment, which enables alarm system designers to use this type of thresholding in a system with complicated and dynamic behavior where simple threshold does not work appropriately.

2) The proposed method is based on a semi-Markov process that has a potential to consider systems with nonGaussian distribution and non-IID behavior. Also, Markov model of n-sample delay can be constructed with less state by the use of SMP theorem.

3) In this paper, the proposed method is combined with GA to tune VTAS parameters and minimize its outcomes such as MAR, FAR, and AAD in a presence of n-sample delay filter. Moreover, other performance indices such as chattering index and sensitivity can be added to the optimization problem in future research works.

4) The prioritization introduced in this paper is a useful and simple measure that can be applied in industrial cases.

\subsection{Limitation of the Proposed Method}

The proposed method faced the following constraints. We aim to resolve all these contraints in our future works.

1) As seen in example 3, the VTAS faces a challenge to separate the normal and abnormal measurements to reduce FAR and MAR. However, by the use of EWMA filter, this problem can be solved.

2) The proposed method addressed the univariate solution of VTAS and it can be extended for multivariate VTAS in the future.

\section{CONCLUSION AND FUTURE WORKS}

Variable threshold adaptive alarm systems are widely used in many industries to reduce alarm nuisance. Performance assessment of such an alarm system is a challenging task and we mention that in the literature there is lack of approaches for performance evaluation of VTAS.. In this study, two most important performance indices for VTAS such as the rate of missed and false alarm was introduced first.. Subsequently, modeling capability of PAND gate has been utilized for the unique and systematic modeling of MAR and FAR. Analytical SMP-based and numerical solutions have been proposed for the evaluation of the MAR and FAR models, which is extendable for different kind of VTAS with and without simple and variable deadbands. Performance evaluation of alarm systems with non-Gaussian characteristic was provided through the use of the semi-Markov process. Furthermore, this study combines the proposed methodology with a genetic algorithm to obtain an optimal selection of parameters. Three numerical examples were provided to depict the effectiveness and capabilities of the proposed method and to compare the results with related research works. The proposed method was also applied to a practical system such asV94-2 Gas turbine.

In the current study we consider univariate VTAS and assumed the measurements as Independent and Identically Distributed (IID). In the future, we have the plan to perform further research to extend this work for multivariate or non-IID VTAS. In this study optimization has been built based on MAR, FAR and AAD in the variety of n-samples of delay-timer, and parameters of adaptive threshold system. In the future, other factors such as chattering index and sensitivity can be considered as optimization goal in addition to other used performance indices.

\section{ACKNOWLEDGMENT}

We thank Professor Enrico Zio from Energy Department, Polytechnic University of Milan, Italy for comments that greatly improved the manuscript. Also, this work was supported by the Noavaran Electronic Adar Sameh company [Grant NO: IRAM17S1].

\section{REFERENCES}

[1] I. Izadi, S. Shah, D. Shook and T. Chen, "An Introduction to Alarm Analysis and Design," in 7th IFAC Symposium on Fault Detection, Supervision and Safety of Technical Processes, Barcelona, Spain, 2009.

[2] N. A. Adnan, Y. Cheng, I. Izadi and T. Chen, "Study of Generalized Delay-Timers in Alarm Configuration," Journal of Process Control, vol. 23, no. 3, p. 382- 395, March 2013.

[3] J. Wang and T. Chen, "An Online Method to Remove Chattering and Repeating Alarms Based on Alarm Durations and Intervals," Computers and Chemical Engineering, vol. 67, no. 7, pp. 43-52, August 2014.

[4] H. Zang, F. Yang and D. Huang, "Design and Analysis of Improved Alarm Delay-Timers," 9th IFAC Symposium on Advanced Control of Chemical Processes, vol. 48, no. 8, p. 669-674, June 2015. 
[5] EEMUA, "Alarm systems: a guide to design, management and procurement," London, 2013.

[6] ISA, "Management of Alarm Systems for the Process Industries," North Carolina, 2009.

[7] J. Xu, J. Wang, I. Izadi and T. Chen, "Performance Assessment and Design for Univariate Alarm Systems Based on FAR, MAR, and AAD," IEEE Transactions on Automation Science and Engineering, vol. 9, no. 2, pp. 296 - 307, December 2011.

[8] P. Goel, A. Datta and M. S. Mannan, "Industrial Alarm Systems: Challenges and Opportunities,” Journal of Loss Prevention in the Process Industries, vol. 50, no. 1, pp. 23-36, 2017.

[9] V. Sharma, A. S. Varma, A. Singh, D. Singh and B. P. Yadav, "A Critical Review on the Application and Problems Caused by False Alarms," in Intelligent Communication, Control and Devices, Singapore, 2018.

[10] A. W. Al-Dabbagh, W. Hu, S. Lai, T. Chen and S. L. Shah, "Toward the Advancement of Decision Support Tools for Industrial Facilities: Addressing Operation Metrics, Visualization Plots, and Alarm Floods," IEEE Transactions on Automation Science and Engineering, vol. 99, no. 1, pp. 1-14, 2018.

[11] M. Quiñones-Grueiro, A. Prieto-Moreno, C. Verde and O. Llanes-Santiago, "Data-Driven Monitoring of Multimode Continuous Processes: A Review," Chemometrics and Intelligent Laboratory Systems, vol. 189, no. 1, pp. 56-71, 2019.

[12] B. R. Hollifield, E. Habibi and J. Pinto, Alarm Management: A Comprehensive Guide, International Society of Automation (ISA), 2011.

[13] S. R. Kondaveeti, S. L. Shah and I. Izadi, "Application of Multivariate Statistics for Efficient Alarm Generation," in 7th IFAC Symposium on Fault Detection, Supervision and Safety of Technical Processes, Barcelona, 2009.

[14] Y. Cheng, I. Izadi and T. Chen, "Optimal Alarm Signal Processing: Filter Design and Performance Analysis," IEEE Transactions on Automation Science and Engineering, vol. 10, no. 2, pp. 446 - 451, April 2013.

[15] F. Yang, S. L. Shah, D. Xiao and T. Chen, "Improved Correlation Analysis and Visualization of Industrial Alarm Data," ISA Transactions, vol. 51, no. 4, pp. 499-506, 2012.

[16] Z. Hao and L. Hongguang, "Optimization of Process Alarm Thresholds: A Multidimensional Kernel Density Estimation Approach," Process Safety Progress, vol. 33, no. 3, p. 292-298, September 2014.

[17] L. Han, H. Gao, Y. Xu and Q. Zhu, "Combining FAP, MAP and Correlation Analysis for Multivariate Alarm Thresholds Optimization in Industrial Process," Journal of Loss Prevention in the Process Industries, vol. 40, no. 2, pp. 471-478, March 2016.

[18] X. Xu, S. Li, X. Song, C. Wen and D. Xu, "The Optimal Design of Industrial Alarm Systems Based on Evidence Theory," Control Engineering Practice, vol. 46, no. 1, p. 142-156, January 2016.

[19] H. Gao, F. Liu and Q. Zhu, "A Correlation Consistency Based Multivariate Alarm Thresholds Optimization Approach,” ISA Transactions, vol. 65, no. 1, pp. 37-43, 2016.

[20] M. Bahar-Gogani, K. Aslansefat and M. A. Shoorehdeli, “A Novel Extended Adaptive Thresholding for Industrial Alarm Systems," in IEEE Iranian Conference on Electrical Engineering, Tehran, Iran, 2017.

[21] J. Taheri-Kalani, K. Aslansefat and G. Latif-Shabgahi, "A Systematic Approach to Design and Analysis of Univariate Alarm Systems Using Penalty Approaches,” Journal of Control, vol. 10, no. 4, pp. 1-15, 2017.

[22] J. Taheri-Kalani, G. Latif-Shabgahi and M. A. Shooredeli, “On The Use of Penalty Approach for Design and Analysis of Univariate Alarm Systems,” Journal of Process Control, vol. 69, no. 1, pp. 103-113, 2018.

[23] H. Alikhani, M. A. Shoorehdeli and M. Yari, "Alarm Management Based Fault Diagnosis of V94. 2 Gas Turbines by Applying Linear Filters," in IEEE 4th International Conference on Robotics and Mechatronics (ICROM), Tehran, Iran, 2016.

[24] M. S. Afzal and T. Chen, "Analysis and Design of Multimode Delay-timers," Chemical Engineering Research and Design, vol. 120, no. 1, pp. 179-193, 2017.

[25] M. S. Afzal, T. Chen, A. Bandehkhoda and I. Izadi, "Analysis and Design of Time-Deadbands for Univariate Alarm Systems," Control Engineering Practice, vol. 71, no. 1, pp. 96-107, 2018.

[26] J. Su, C. Guo, H. Zang, F. Yang, D. Huang, X. Gao and Y. Zhao, "A Multi-Setpoint Delay-timer Alarming Strategy for Industrial Alarm Monitoring," Journal of Loss Prevention in the Process Industries, vol. 54, no. 1, pp. 1-9, 2018.

[27] S. Cai, A. Palazoglu, L. Zhang and J. Hu, "Process Alarm Prediction Using Deep Learning and Word Embedding Methods," ISA Transactions, pp. In Press, Corrected Proof, 2018.

[28] Y. Sun, W. Tan and T. Chen, “A Method to Remove Chattering Alarms Using Median Filters," ISA Transactions, vol. 73, no. 1, pp. 201-207, 2018 .

[29] J. B. Fussell, E. F. Aber and R. G. Rahl, “On the Quantitative Analysis of Priority-AND Failure Logic,” EEE Transactions on Reliability, 25(5), , vol. 25, no. 5, pp. 324-326, 1976.

[30] M. Mitchell, An Introduction to Genetic Algorithms, USA: MIT Press, 1998.

[31] M. Witczak, M. Mrugalski and J. Korbicz, "Towards Robust Neural Network-based Sensor and Actuator Fault Diagnosis: Application to a Tunnel Furnace," Neural Processing Letters, vol. 42, no. 1, pp. 17-87, 2015. 
[32] E. Naghoosi, "Alarm Limits, Deadbands and Chattering (Master Thesis)," 2009.

[33] A. Hugo, "Estimation of Alarm Deadbands," in 7th IFAC Symposium on Fault Detection, Supervision and Safety of Technical Processes, Barcelona, 2009.

[34] E. Naghoosi, I. Izadi and T. Chen, "A Study on the Relation between Alarm Deadbands andOptimal Alarm Limits," in American Control Conference, San Francisco, 2011.

[35] S. M. de-Oca, V. Puig and J. Blesa, "Robust fault detection based on adaptive threshold generation using interval LPV observers," International Journal of Adaptive Control and Signal Processing, vol. 26, no. 3, pp. 258-283, July 2012.

[36] K. Patan, Artificial Neural Networks for the Modelling and Fault Diagnosis of Technical Processes, Berlin, Germany: Springer, 2008.

[37] J. B. Dugan, S. J. Bavuso and M. A. Boyd, "Dynamic Fault Tree Models for Fault-Tolerant Computer Systems," IEEE Transactions on Reliability, vol. 41, no. 3, pp. 363-377, 1992.

[38] G. Merle, J. M. Roussel, J. J. Lesage and A. Bobbio, "Probabilistic Algebraic Analysis of Fault Trees With Priority Dynamic Gates and Repeated Events," IEEE Transactions on Reliability, 59(1), pp-250., vol. 59, no. 1, pp. 250-261, 2010.

[39] H. Boudali and J. Bechta Dugan, "A Discrete-Time Bayesian Network Reliability Modeling and Analysis Framework," Reliability Engineering and System Safety, vol. 87, no. 3, p. 337-349, 2005.

[40] D. Codetta-Raiteri, "The Conversion of Dynamic Fault Trees to Stochastic Petri Nets, As a Case of Graph Transformation," Electronic Notes in Theoretical Computer Science, vol. 127, no. 2, pp. 45-60, 2005.

[41] S. Kabir, M. Walker and Y. Papadopoulos, "Dynamic system safety analysis in HiP-HOPS with Petri Nets and Bayesian Networks," Safety Science, vol. 105, no. 1, pp. 55-70, 2018.

[42] M. Zajac and A. Kierzkowski, "Attempts at Calculating Chosen Contributors with Regard to the Semi-Markov Process and the Weibull Function Distribution," in Summer Safety and Reliability Seminars (SSARS), Gdañsk-Sopot, Poland, 2011.

[43] V. G. Kulkarni, Modelling and Analysis of Stochastic Systems, London, UK: Chapman \& Hall, 1995.

[44] L. Yin, R. M. Fricks and K. Trivedi, "Application of Semi-Markov Process and CTMC to Evaluation of UPS System Availability," in Annual Reliability and Maintainability Symposium, Seattle, WA, 2002.

[45] F. Frattini, A. Bovenzi, J. Alonso and K. Trivedi, "Reliability Indices," in Wiley Encyclopedia of Operations Research and Management Science, John Wiley \& Sons, Inc., Hoboken, NJ, 2013, pp. 1-21.

[46] R. Fricks, M. Telek, A. Puliafito and K. Trivedi, "Markov Renewal Theory Applied to Performability Evaluation," in Stateof-the-art in Performance Modeling and Simulation. Modeling and Simulation of Advanced Computer Systems: Applications and Systems, Newark, NJ, Gordon \& Breach, 1998, pp. 193-236.

[47] D. Rios Insua, F. Ruggeri and M. P. Wiper, "Appendix B: Generating Functions and the Laplace-Stieltjes Transform," in Bayesian Analysis of Stochastic Process Models, Chichester, UK. doi: 10.1002/9780470975916.app2, John Wiley \& Sons, Ltd, 2012, pp. 283-284.

[48] M. Zajac and T. Budny, "On Determination of Some Characteristics of Semi-Markov Process for Different Distributions of Transient Probabilities," Journal of Reliability \& Risk Analysis: Theory \& Applications, vol. 2, no. 1, pp. 74-84, 2009.

[49] S. Distefano, F. Longo and K. S. Trivedi, "Investigating Dynamic Reliability and Availability through State-Space Models," Computers \& Mathematics with Applications, vol. 64, no. 12, pp. 3701-3716, December 2012.

[50] R. Manian, D. W. Coppit, K. J. Sullivan and J. Bechta Dugan, "Bridging the Gap Between Systems and Dynamic Fault Tree Models," in IEEE Reliability and Maintainability Symposium (RAMS), Washington, DC, 1999.

[51] J. Bechta Dugan, S. J. Bavuso and M. A. Boyd, "Fault Trees and Markov Models for Reliability Analysis of Fault-Tolerant Digital Systems," Reliability Engineering \& System Safety, vol. 39, no. 3, pp. 291-307, 1993.

[52] P. Zhu, J. Han, L. Liu and M. Zuo, "A Stochastic Approach for the Analysis of Fault Trees With Priority AND Gates," IEEE Transactions on Reliability, vol. 63, no. 2, pp. 480 - 494, April 2014.

[53] N. Adnan, I. Izadi and T. Chen, "On Expected Detection Delays for Alarm Systems with Deadbands and Delay-Timers," Journal of Process Control, vol. 21, no. 9, pp. 1318-1331, October 2011.

[54] G. Bohrenkämper, D. Reiermann, G. Höhne and U. Lingner, "Technology Evolution of the Proven Gas Turbine Models V94. 2 and V84. 2 for New UNITS and Service Retrofits," Siemens Report, Germany, 2004.

[55] P. Barbarini, G. Franzoni and E. Kulot, "Alarm Prioritization at Plant Design Stage-A Simplified Approach," London, Taylor \& Francis Group, 2009. 